\title{
Characteristics of the method of creating innovative raw materials for the production of carbon fibers on the basis of coal from the Kuznetsk Basin
}

\author{
E.V. Vasilyeva, I.S. Vetoshkina,T.G. Cherkasova, S.P. Subbotin, A.V. Nevedrov, A.V. Papin, \\ V.S. Solodov \\ T.F. Gorbachev Kuzbass State Technical University,Department Chemical Technology of Solid Fuel \\ Institute of Chemicaland Oil-Gaz Technologies, Kemerovo, Russian Federation,
}

\begin{abstract}
Studies on thermal dissolution of $\mathrm{G}, \mathrm{GZh}$ and $\mathrm{Zh}$ coals in anthracene oil were carried out in the laboratory of PAO Koks together with the Department of Solid Fuel Chemical Technology of the Institute of chemical and oil and gas technologies. The optimal parameters of the process were determined, and the quality of the obtained products was studied. The obtained indicators are compared with the quality indicators of raw materials required for the production of carbon fibers. It is shown that it is possible to obtain raw materials directly from coal, bypassing the high-temperature coking process, for the production of a binding material with the required properties.
\end{abstract}

\section{Carbon fiber and the relevance of its production}

The development of various fields of science and technology, the emegence of higher requirements for the properties of products, the tightening of environmental standards, all this contributes to an increase in the consumption of carbon fibers and products based on them. The extensive use of carbon fiber-based materials allows not only to obtain products that are unique in their properties and are used in various fields but also to reduce environmental pollution by emissions of carbon dioxide and savemanytons. Over the period from 2008 to 2013, the consumption of carbon fibers in the world increased almost 1.6 times and reached 48 thousand tons.According to forecasts of experts from Carbon Composites e. V., by 2020, this figure may already be about 130 thousand tons. Growing demand pushes manufacturers to expand their capacities; this applies to both traditional market players and new manufacturers. The countries of the Customs Union are trying to keep up with global trends, but they are still in the last positions of the ranking, occupying only $1.6 \%$ of the world market.

Carbon fiber is a nanostructured organic material that contains $92-99.99 \%$ carbon and has high values of strength and modulus of elasticity. Carbon fiber is obtained by hightemperature transformations without air access (pyrolysis process) from polymer fibers (precursors), which give the highest yield of carbon residue during pyrolysis. Their structural and chemical features completely determine the technology used. 
Polyacrylonitrile (PAN) fibers, conventional and liquid-crystal (mesophase) pitches, viscose filaments, hydrate cellulose, phenol-formaldehyde resin, etc. can be used as precursors. Depending on the nature of the precursor and production modes, carbon fibers with different properties are obtained: high-strength or high-modulus fibers with increased strength and elongation, as well as general-purpose multi-purpose carbon fibers (Table 1) [1].

From the analysis of Table 1, it can be concluded that pitch-based fibers have high values of strength and elastic modulus and are the most expensive, but in the Russian Federation, due to the lack of high-quality raw materials and proven production technologies, they are not produced.

Table 1. Comparison of carbon fiber types.

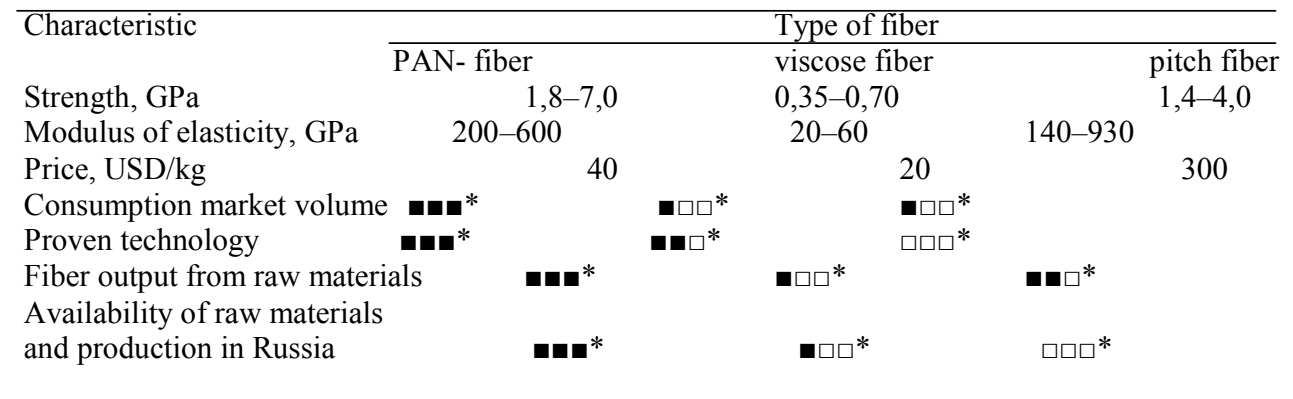

*ח - presence of the attribute. Designation of the indicator degree: $\mathbf{m} \mathbf{m}-$ high; $\mathbf{m} \square-$ medium; - $\square \square$ - small; $\square \square \square-$ the indicator is missing.

Coal pitch is associated with considerable environmental impact, due to its high content of aromatic polycyclic hydrocarbons, Including benzo[ $a]$ pyrene. Accordingly, the development of lower-impact methods of raw materials for the production of carbon fiber is of great importance [2]. At present, the production of alternative pitch products is based on petroleum, whose benzo[a]pyrene content is much lower than that of coal pitch. However, petroleum pitch is characterized by high sulfur content and low yield of coke residue.

The most promising alternative to coal pitch is the binder produced by the thermal solution of coal, without traditional coking. [2]. The temperature selected for the thermal solution of coal corresponds to the onset of thermal decomposition of the coal. The resulting free radicals enter into chemical interaction with the solvent. That slows secondary polycondensation, and soluble reaction products accumulate [3]. The anthracene fraction of coal tar is an effective solvent, which is chemically similar to the solution products. The effectiveness of anthracene oil is related to the presence of active hydrogen donors (acenaphthene, dihydroanthracene, fluorene, and carbazole), molecules transferring hydrogen (phenanthrene, fluoranthene), and compounds with solvating properties (quinoline, indole). The residue contains polycondensed aromatic hydrocarbons and may serve as a replacement for coal pitch. Analysis of research data indicates that the best raw materials are the anthracene fraction of coal tar and coal of moderate metamorphic development with a considerable plastic layer thickness $(y)$ [4].

The purpose of the study is to investigatethe possibility of obtaining raw materials for the production of carbon fibers by thermal dissolution of coals.

The research objectives are development of technology for producing extractive pitch based on coal from the Kuznetsk basin; study of the quality of the resulting peck-like product; study of the possibility of producing carbon fibers from the extractive pitch. 


\section{Materials and methods}

Due to the urgency of the problem of shortage of high-quality raw materials for the production of carbon fibers, research conducted in the laboratory of PAO Koks together with the Department of Solid Fuel Chemical Technology of the Institute of Chemical and Oil and Gas Technologies makes possible the production of an alternative binder by dissolving coal in anthracene oil. The thermal solution of coal is carried out in experimental apparatus with a sealed reactor, in which the temperature and the heating rate may be adjusted. To measure the pressure in the reactor, a pressure gauge is attached to the tube in its lid. The excess gaseous products are released using a ball valve through a hydraulic trap. In Fig. 1, we show the basic apparatus.

The raw materials in the research are anthracene oil corresponding to State Standard GOST 11126-88; and G, GZh, and Zh coal concentrates used in coking batch at PAO Koks. Tables 2 and 3 present the quality of the initial coal concentrates and the anthracene oil, respectively. The working moisture of coals was determined according to ISO 589:2008, ash content according to ISO 1171:2010, yield of volatile substances according to ISO 562:2010, dilatometric indicator $\mathrm{I}_{\mathrm{b}}$ according to State Standard GOST 14056-77, plastometric indicators (y, x) according to State Standard GOST 1186-2014, petrographic indicators according to ISO 7404-5:2009.

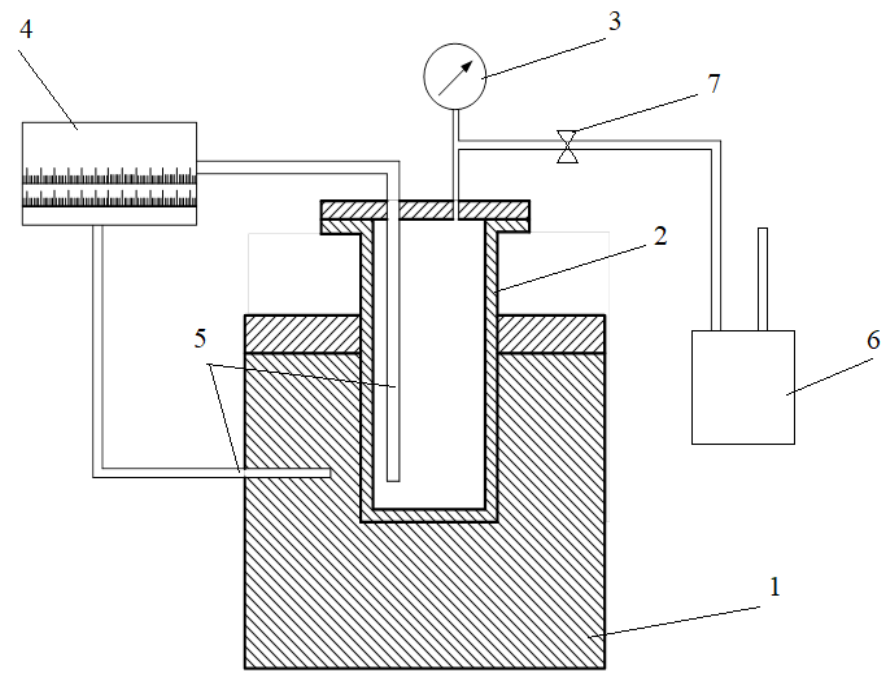

Fig. 1. Apparatus for the thermal solution of coal: 1 - shaft-type electrofurnace; 2 - reactor; 3 manometer; 4 - monitoring instruments; 5 -thermocouples; 6 - hydraulic trap; 7 -ball valve for the release of excess pressure.

Table 2. Characteristics of coal concentrates.

\begin{tabular}{|c|c|c|c|c|c|c|c|c|}
\hline \multirow[t]{2}{*}{ Coal rank } & $\mathrm{W}_{\mathrm{t}}^{\mathrm{r}}$ & $A^{d}$ & $\mathrm{~V}^{\mathrm{d}}$ & $\mathrm{I}_{\mathrm{b}}$ & $\mathrm{y}$ & $\mathrm{x}$ & $\mathrm{R}_{0}$ & $\mathrm{Vt}$ \\
\hline & $\%$ & $\%$ & $\%$ & $\mathrm{~mm}$ & $\mathrm{~mm}$ & $\mathrm{~mm}$ & $\%$ & $\%$ \\
\hline G & 10.4 & 7.9 & 38.2 & 123 & 18 & 42 & 0.685 & 95.0 \\
\hline
\end{tabular}




$\begin{array}{lcccccccc}\text { Zh } & 7.5 & 8.9 & 29.8 & 154 & 34 & 3 & 0.918 & 87.0 \\ \text { GZh } & 9.1 & 8.5 & 33.9 & 132 & 19 & 42 & 0.799 & 92.0\end{array}$

Table 3. Characteristics of anthracene oil.

$\begin{array}{lll}\text { Characteristic Value } & \end{array}$

Density at $20{ }^{\circ} \mathrm{C}, \mathrm{kg} / \mathrm{m}^{3}$

Water content, wt \%

1130

Content of toluene-insoluble materials, wt $\%$

Ash content, $\%$

Components, \%:

naphthalene

0.02

$\beta$ - naphthalene

$\alpha$ - naphthalene

dimethylnaphthalene

acenaphthtene

diphenylene oxide

fluorene

anthracene

To determine the optimal conditions of the thermal solution of the coal, we conducted experiments to determine the influence of the solution parameters on the product quality. Coal samples for research were taken from batches of fuel received by the company in accordance with GOST 10742-71. The coal concentrates are crushed using a jaw crusher Retsch $\mathrm{BB} 50$ to $<1 \mathrm{~mm}$ and dried to $<1 \%$ moisture content in the drying cabinet at a temperature of $100^{\circ} \mathrm{C}$ and mixed in different proportions with anthracene oil. The resulting paste is placed in the reactor chamber. The reactor is placed into a shaft furnace preheated to $300-350^{\circ} \mathrm{C}$. The process takes 1 hour. The pitch-like product is shown in Fig. 2.

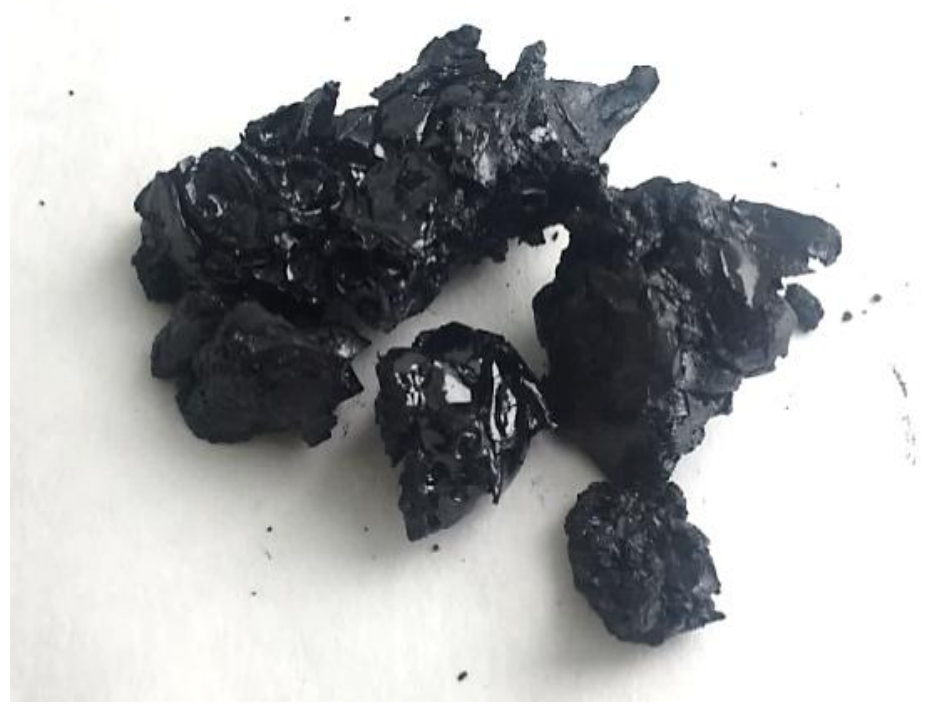

Fig. 2. Pitch-like product. 


\section{Results}

The product quality is assessed on the basis of the properties of the binder pitch itemized in State Standard GOST 10200-2017: the ash content, the yield of volatiles, the content of toluene-insoluble materials, and the content in quinoline-insoluble materials (Table 4).

The key issue in obtaining high-quality fibers is the presence of mesophase in the sand or its ability to transition to the state of mesophase pitch $[5,6]$. To determine the presence of mesophase in the resulting product, studies of its optical activity were performed using a MIN-8 polarization microscope. Optical studies were performed for a sample of extractive pitch obtained by thermal dissolution of GZh coal at a solvent/coal ratio of 60/40 at a temperature of $370^{\circ} \mathrm{C}$. Fig. 3 shows a photo of the peck film in ordinary light, and Fig. 4 shows it in polarized light. From the analysis of microphotography (Fig. 4), it can be seen that the pitch obtained by thermal dissolution of coals is capable of forming a mesophase, but its amount is small. To increase the amount of mesophase, the resulting pitch must be subjected to heat treatment.

Table 4. Influence of the coal rank, the solvent/coal ratio, and the temperature of thermal solution on the product quality

\begin{tabular}{|c|c|c|c|c|c|c|c|c|}
\hline \multicolumn{2}{|c|}{ Coal rankSolvent/coal } & \multirow{2}{*}{$\frac{\mathrm{T}_{\text {reaction }}}{{ }^{\mathrm{O}} \mathrm{C}}$} & \multicolumn{2}{|c|}{ Product yield } & \multirow{2}{*}{$\frac{\mathrm{T}_{\mathrm{so}}}{{ }^{{ }^{\circ} \mathrm{C}}}$} & \multirow{2}{*}{$\frac{\mathrm{A}^{\mathrm{d}}}{\%}$} & $V^{d} n / t^{*}$ & \multirow{2}{*}{$\begin{array}{c}\mathrm{n} / \mathrm{x}^{* *} \\
\%\end{array}$} \\
\hline & & & & $\overline{\%}$ & & & $\% \quad \%$ & \\
\hline G & $70 / 30$ & 350 & 69.4 & 58 & 2.1 & 76.4 & 38.7 & 6.2 \\
\hline G & $70 / 30$ & 370 & 70.3 & 70 & 2.5 & 74.2 & 38.9 & 5.8 \\
\hline G & $70 / 30$ & 400 & 71.6 & 65 & 1.5 & 76.4 & 32.7 & 3.9 \\
\hline G & $60 / 40$ & 370 & 73.1 & 125 & 1.9 & 56.5 & 39.3 & 5.8 \\
\hline G & $60 / 40$ & 400 & 74.1 & 127 & 2.5 & 57.2 & 41.3 & 6.5 \\
\hline G & $50 / 50$ & 370 & 86.6 & 135 & 2.7 & 64.4 & 42.9 & 5.6 \\
\hline G & $50 / 50$ & 400 & 82.2 & 138 & 2.3 & 60.9 & 45.2 & 23.1 \\
\hline G & $80 / 20$ & 370 & 80.1 & $<130$ & 1.2 & 85.2 & 19.5 & 1.9 \\
\hline $\mathrm{Zh}$ & $70 / 30$ & 370 & 72.0 & 58 & 1.7 & 70.7 & 29.2 & 8.4 \\
\hline $\mathrm{Zh}$ & $70 / 30$ & 390 & 71.5 & 61 & 1.4 & 71.0 & 26.2 & 7.2 \\
\hline $\mathrm{Zh}$ & $60 / 40$ & 370 & 74.8 & 117 & 2.7 & 60.7 & 41.5 & 9.1 \\
\hline $\mathrm{Zh}$ & $60 / 40$ & 390 & 73.5 & 127 & 2.6 & 64.9 & 41.2 & 24.8 \\
\hline GZh & $70 / 30$ & 370 & 73.4 & 69 & 2.0 & 71.3 & 29.5 & 5.8 \\
\hline GZh & $70 / 30$ & 390 & 71.5 & 63 & 1.4 & 73.8 & 27.6 & 4.0 \\
\hline GZh & $60 / 40$ & 370 & 75.2 & 110 & 1.9 & 57.0 & 41.2 & 6.8 \\
\hline GZh & $60 / 40$ & 390 & 75.9 & 126 & 3.8 & 57.1 & 58.3 & 26.2 \\
\hline
\end{tabular}

$*_{\mathrm{n}} / \mathrm{t}$-content of toluene-insoluble materials ( $\alpha$ fraction);

$* * \mathrm{n} / \mathrm{x}$ - content of quinoline-insoluble materials ( $\alpha_{1}$ fraction). 


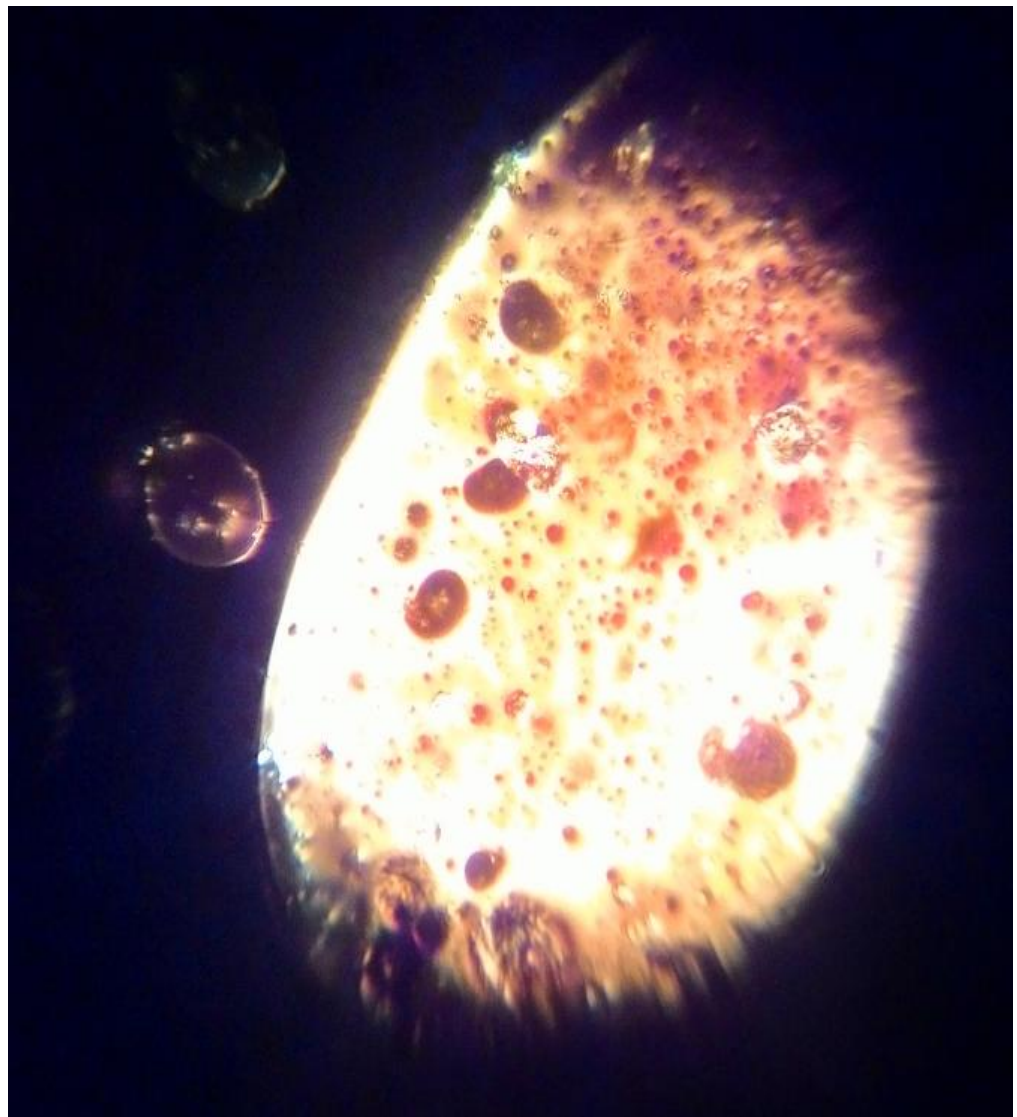

Fig. 3. The appearance of the pitch film in passing ordinary light. 


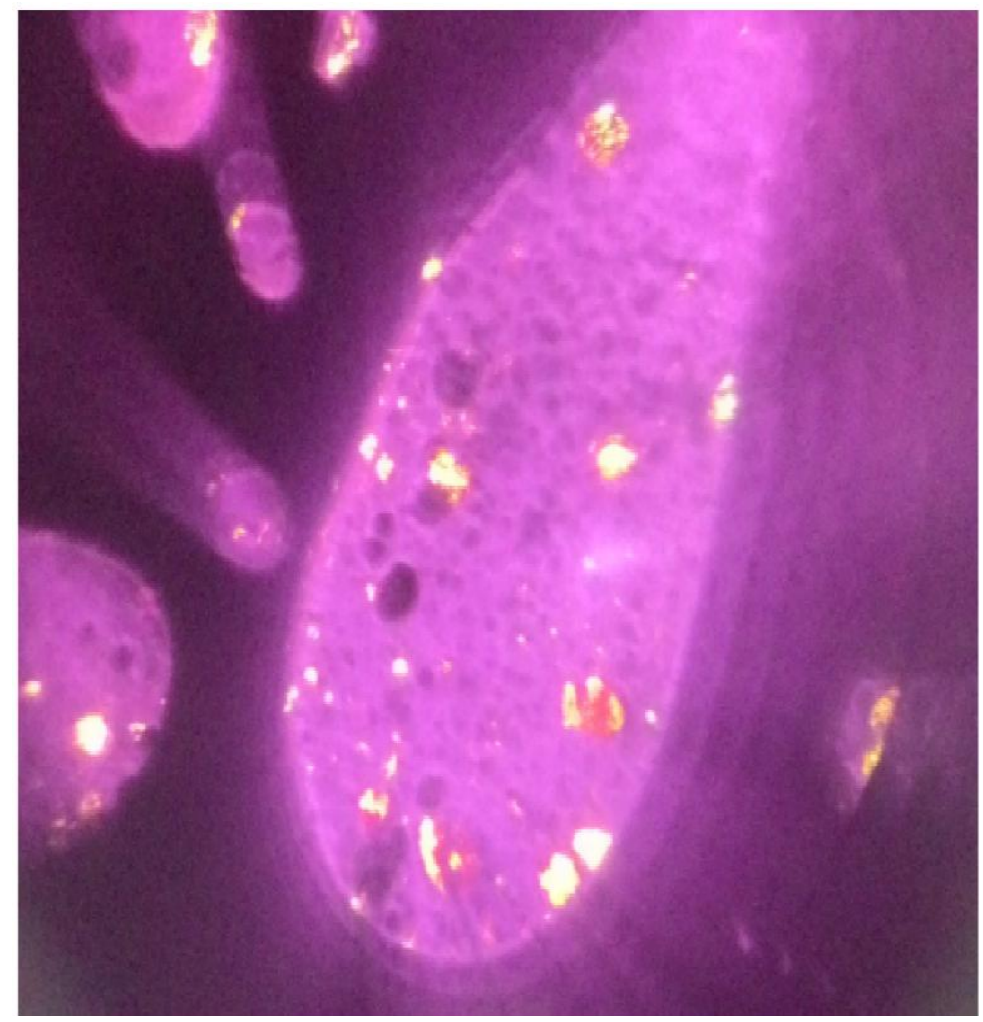

Fig. 4. The appearance of extractive pitch film in polarized light.

\section{Conclusions}

From the analysis of the research results, it can be concluded that the optimal conditions for obtaining a thermally dissolved product with a low softening point can be achieved with a solvent/coal ratio of $70 / 30$ and a reaction temperatureof $390-400^{\circ} \mathrm{C}$. This pitch is characterized by the lowest ash content and the lowest content of $\alpha$ and $\alpha_{1}$ fractions. However, in this case, the softening temperature does not correspond to this indicator for fiber-forming pitches, so it is necessary to further process it to increase the softening temperature and reduce the yield of volatile substances.

To obtain a product with a high softening temperature, the optimal conditions are the ratio of solvent/coal $-60 / 40$, the temperature in the reactor $-370^{\circ} \mathrm{C}$. However, even in this case, further processing is necessary to increase the softening temperature and reduce the yield of volatile substances.

Particularly worth noting is the high ash content of samples, which determines a separate layer of research to reduce this indicator.

Thus, to obtain raw materials for the production of fibers, the product of thermal dissolution of coal obtained in the course of research shouldbe further purified from excessive amounts of mineral impurities, which will reduce ash content and oxidation process to increase the softening temperature. However, the resulting pitch with a reduced content of polycyclic aromatic hydrocarbons without additional purification can be used in technologies that do not impose high requirements on ash content (for example, the production of carbon refractories and refractory masses). 


\section{References}

1. S. Kim, Raw materials-composites-carbon fiber. The Chemical Journal 10: 64-73 (2014).

2. E.N. Marakushina, Preparation of pitches and binders by the thermal dissolution of coals.(Krasnoyarsk: SFU,2015).

3. P.N. Kuznecov, N.V. Perminov, Thermal dissolution of coal in technical pasteurizers and their mixtures. Coke and chemistry11: 16-23. (2019).

4. A.E. Bazegskij, M.B. Shkoller, Investigation of the process of thermal dissolution of coal in order to obtain a binder for refractory masses. News of higher education institutions: iron and steel industry,59(8): 517-522(2016).

5. V.E. Privalov, Coal tar pitch.(Moscow: Metallurgiya,1981).

6. I.S. Vetoshkina, V.S. Solodov, Thermal dissolution of coals of the raw material base of PAO «Koks» in the medium of anthracene fraction of coal tar. Coke and chemistry2: 51-54(2019). 